lower, indicating suboptimal use of space and highlighting an opportunity to redesign and improve functionality.

Many short-stay patients were admitted to the other wards, whilst longer-stay patients were admitted onto PSSU. Particular breeches in the PED were felt to have been avoidable if shortstay beds had been available.

Although patient experience was positive, feedback from the staff emphasised the frustration of working in such a chaotic environment.

The information was fed back to the Paediatric Executive Board.

Effects of changes The PSSU is reclaiming it's short-stay status. Nurse-led discharge has been introduced and admission pathways from the PED redesigned to improve patient flow. A 'virtual PSSU' trial is underway on the ward, with ring-fencing of a number of beds to be reserved for short stay patients and to be staffed by PSSU. Relevant workload has been diverted to outpatients and a merger of PSSU and surgical day unit is being considered. Modelling of very short-stay patients suitable for a colocated observation bay, is informing plans for the PED rebuild. Lessons learnt Initially the 'PSSU problem' seemed too complicated to solve. Working with designers in the QI Sprint allowed us to devise a novel approach to improving the quality of care provided within the PSSU. The data gathering exercise was very powerful and quantified the issues objectively. This enabled us to devise a clear message when disseminating findings and campaigning for change. Mapping individual patient journeys brought a human face to the unit.

Message for others Data is powerful and can help define an 'undefinable' problem.

\section{G530(P) TO GIVE OXYGEN OR NOT? ARE WE ADHERING TO LOCAL GUIDELINES ON ADMINISTERING TARGETED OXYGEN THERAPY TO OUR NEONATAL POPULATION?}

${ }^{1} \mathrm{C}$ Willis, ${ }^{2} \mathrm{~K}$ Houston. ${ }^{1}$ Paediatrics, NHS, Glasgow, UK; ${ }^{2}$ Neonates, NHS, Glasgow, UK

\subsection{6/archdischild-2015-308599.481}

Context This "spot audit" was carried out in a level 3 neonatal unit.

Problem Supplementary oxygen therapy is a vitial to the vulnerable neonate. We know that in excess, oxygen can be toxic, contributing to retinopathy of prematurity (ROP). The recent BOOST 2 study has made clinicians rethink what our oxygen saturation limits should be. However, given the increased mortality in those with lower saturation limits, the exact limit remains controversial.

The aim of this "spot audit" was simply to determine whether we were adhering to our local guidelines regarding oxygen saturation targeting.

Assessment of problem and analysis of its causes This audit was carried out on a weekly basis. The initial few weeks, before any change, ensured that we obtained baseline numbers. The staff were aware that the audit project was being carried out.

We quickly identified that there was a need to intervene and a discussion was had with ward sisters. We identified that nurses were key to ensuring that the limits on the saturation monitors were set correctly.

Intervention We realised that verbal communication/education alone would be insufficient to increase our compliance. A visual aid, or quick reference card, was developed. This briefly summarised our protocol, i.e. what the saturation limits should be for neonates, based on their risk of ROP. This was produced in a size which ensured that it could be attached to the saturation monitors.

Study design A initial prospective audit was carried out over a period of 8 weeks. information regarding risk of ROP and whether or not stauration limits were achieved was collected on a proforma. When possible, I would then refer to the neonates case notes and/or speak to nursing staff caring for them to determine why the monitors may have been set differently to protocol, i.e. medical decision or in error.

2 years after the initial audit, we've reaudited the same thing to determine if compliance has been maintained.

Strategy for change Initially discussions were had with the ward sister regarding the audit project, but it became apparent that there was a lack of awareness of our local protocol. The quick reference card was produced on a home computer. Medical physics (who ensure up keep of our saturation monitors) were given additional copies of the visual aid. I presented the inital results at a local quality improvement evening.

Measurement of improvement Percentages were used to demonstrate compliance. This ensured that all staff could easily interpret the results obtained. During the initial four weeks of the audit, our compliance with our local guideline, regarding oxygen saturation targets within the neonatal population, ranged from 43-70\%. After the introduction of the quick reference card, our compliance was 79-94\%. We re-audited this recently and our compliance is sustained at $92 \%$. In the majority of cases, the reasons for non compliance included no quick reference card on the saturation monitor!

Effects of changes Our change has increased compliance with local guidelines and ensures that oxygen therapy is being targeted appropriately in the majority of cases. It has also increased staffs awareness of the importance of targeted oxygen therapy.

Lessons learnt This simple regular audit process can be applied within any healthcare setting. The simplicity of the concept makes it easily reproducibile. Furthermore, it helped identify a key, often overlooked, problem within our unit, and attempted to address it.

Message for others We have demonstrated how simple auditing can result in sustained improvement in neonatal care by targeting our oxygen saturations more effectively in compliance with our local guideline.

\section{G531(P) IMPROVING THE SAFETY AND QUALITY OF HANDOVER}

ST Williams, K Pryde, V Vijay, V Irvine, F Hignett. Southampton Children's Hospital, University Hospital Southampton, NHS Trust, Southampton, UK

\subsection{6/archdischild-2015-308599.482}

Context This project aimed to improve handover for paediatric medical specialties, paediatric surgery and general paediatrics at a regional tertiary paediatric centre. As well as doctors of varying grade and specialty, others involved include the nursing outreach team and bed manager.

Problem Handover occurs three times a day and with 50-80 complex patients it needs to be an efficient process ensuring patient safety and communication of essential appropriate information. Issues around the quality and safety of handover were highlighted from clinicians within the department, trainee feedback and the GMC survey.

Assessment of problem and analysis of its causes Baseline measurements were obtained over 16 consecutive handovers. 